\title{
Multi-coloured Hamilton cycles in random edge-coloured graphs
}

\author{
Colin Cooper* $\quad$ Alan Frieze ${ }^{\dagger}$
}

June 11, 2001

\begin{abstract}
We define a space of random edge-coloured graphs $\mathcal{G}_{n, m, \kappa}$ which correspond naturally to edge $\kappa$-colourings of $G_{n, m}$. We show that there exist constants $K_{0}, K_{1} \leq 21$ such that provided $m \geq K_{0} n \log n$ and $\kappa \geq K_{1} n$ then a random edge coloured graph contains a multi-coloured Hamilton cycle with probability tending to 1 , as the number of vertices $n$ tends to infinity.
\end{abstract}

\section{Introduction}

Let $\mathcal{G}_{n, m, \kappa}$ denote the space of random edge-coloured graphs, defined as follows: Each $G$ in $\mathcal{G}_{n, m, \kappa}$ has vertex set $[n]$, edge set $E_{m}(G)$ of size $m$, and each edge is coloured with a label from $[\kappa]$. Thus $\left|\mathcal{G}_{n, m, \kappa}\right|=\left(\begin{array}{l}N \\ m\end{array}\right) \kappa^{m}$ where $N=\left(\begin{array}{l}n \\ 2\end{array}\right)$. The elements of $\mathcal{G}_{n, m, \kappa}$ are given the uniform measure. A random edge-coloured graph $G_{n, m, \kappa}$ is a graph $G$ sampled uniformly at random from $\mathcal{G}_{n, m, \kappa}$.

Given $G \in \mathcal{G}_{n, m, \kappa}$, a subset $S$ of the edges of $G$ is multi-coloured, if no two edges of $S$ have the same colour. We are interested in conditions on $n, m, \kappa$ which imply that $\mathbf{w h p}^{1} G_{n, m, \kappa}$ contains a multi-coloured Hamilton cycle. We also consider the corresponding randomly arc-coloured random digraph $D_{n, m, \kappa}$ which is defined in the analogous way. We prove

${ }^{*}$ School of Mathematical and Computing Sciences, Goldsmiths College, University of London, London SE14 6NW, UK. E-mail: c.cooper@gold.ac.uk.

${ }^{\dagger}$ Department of Mathematical Sciences, Carnegie Mellon University, Pittsburgh PA15213, U.S.A., Supported in part by NSF grant CCR-9818411. E-mail: alan@random.math.cmu.edu.

${ }^{1}$ A sequence of events $\mathcal{E}_{n}$ is said to occur with high probability (whp) if $\lim _{n \rightarrow \infty} \operatorname{Pr}\left(\mathcal{E}_{n}\right)=1$. 
Theorem 1 There exist constants $K_{0}, K_{1}>0$ such that if $m \geq K_{0} n \log n$ and $\kappa \geq K_{1} n$ then $\operatorname{whp} G_{n, m, \kappa}$ (resp. $\left.D_{n, m, \kappa}\right)$ contains a multi-coloured Hamilton cycle.

We note that Theorem 1 is best possible up to a constant factor, for we need at least $n$ distinct colours and the underlying graph (resp. digraph) does not contain a Hamilton cycle whp until $m>n \log n / 2$. The values of the constants, $\left(K_{0}, K_{1}=21\right)$, we use in the proof of Theorem 1 are, however, not the best possible values.

There are two general types of results on multi-coloured structures: whp existence under random colouring and guaranteed existence under adversarial colouring. When considering adversarial (worst-case) colouring, the guaranteed existence of multi-coloured structures, is called an Anti-Ramsey property.

Erdös, Nesetril and Rödl [5], Hahn and Thomassen [8] and Albert, Frieze and Reed [1] (correction in Rue [10]) considered colourings of the edges of the complete graph $K_{n}$ where no colour is used more than $k$ times. It was shown in [1] that if $k \leq n / 64$, then there must be a multi-coloured Hamilton cycle. Cooper and Frieze [3] proved a random graph threshold for this property to hold in almost every graph in the space studied.

With respect to random colouring, Janson and Wormald [9] gave conditions for the existence of a multi-coloured Hamilton cycle in a random regular graph. We also mention that Frieze and Mckay [7] found a tight threshold for the existence of a multicoloured spanning tree.

\section{A sequence of random graphs}

Because we are concerned with monotone properties, we can work entirely with the independent model $G_{n, p, \kappa}$ where $p=m / N$ and the underlying uncoloured graph is $G_{n, p}$. Let $p_{1}$ satisfy $1-p=\left(1-p_{1}\right)^{2}$. Let $D_{n, p_{1}}$ be the random digraph where each arc occurs independently with probability $p_{1}$. Suppose now that we randomly colour the arcs of $D_{n, p_{1}}$ with $\kappa$ colours to obtain the random coloured graph $D_{n, p_{1}, \kappa}$. Ignoring orientation gives us the random graph $G_{n, p, \kappa}$, provided we make a random choice from the two possible colours when coalescing the edges of directed 2-cycles.

Next let $\mathcal{D}_{d-\text { out }, \kappa}$ denote the following set of arc-coloured digraphs: Each $D \in \mathcal{D}_{d-\text { out }, \kappa}$ has vertex set $[n]$, each vertex has out-degree $d$ and the $\operatorname{arcs}$ of $D$ are multi-coloured by $[\kappa]$ i.e. no colour is used more than once. Thus $\left|\mathcal{D}_{d-o u t, \kappa}\right|=\left(\begin{array}{c}n-1 \\ d\end{array}\right)^{n}\left(\begin{array}{c}\kappa \\ d n\end{array}\right)(d n)$ !. The arc-coloured digraph $D_{d-o u t, \kappa}$ is chosen uniformly at random from $\mathcal{D}_{d-o u t, \kappa}$. In this paper we will be concerned with $d=O(1)$, in particular we assume that $d=5$ from now on. 
The central idea of this paper is to use a network flow algorithm to take $D_{n, p_{1}, \kappa}$ and, conditional on an event of probability 1-o(1), return as output, a multi-coloured subdigraph $D$. The distribution of $D$ will be that of $D_{d-o u t, \kappa}$. If we ignore orientation in $D_{d-o u t, \kappa}$ and delete parallel edges then we obtain the random multi-coloured graph $G_{d-o u t, \kappa}$. Ignoring colours now gives us the random graph $G_{d-o u t}$. If it is known whp that $G_{d-\text { out }}$ is Hamiltonian, then we will have proved that whp $G_{n, p, \kappa}$ contains the required multicoloured $H$. To prove Theorem 1 for $G_{n, m, \kappa}$ we only have to do this for $d \geq 5$ and then apply the result of Frieze and Luczak [6] which states that such a graph is Hamiltonian whp. There is a technical point here. In the usual construction of $G_{d-\text { out }}$ we coalesce rather than delete parallel edges. It is not difficult to see that the proof of [6] is easily modifiable to handle this. On the other hand the result of Cooper and Frieze [4] that $G_{4-o u t}$ is Hamiltonian whp seems to run into difficulty.

\section{$2.1 \quad$ Network Flow Construction}

We define a flow network $\mathcal{N}$ as follows. $\mathcal{N}$ has source $s$ and $\operatorname{sink} t$. The vertex set $W$ consists of $s, t$, the set of colours $C=[\kappa]$ and the set $V=[n]$ of vertices of the $D_{n, p_{1}, \kappa}$ under consideration. For each colour $x \in C$ there is an $\operatorname{arc}(s, x)$ in $\mathcal{N}$ of capacity 1. There is an arc $(x, v)$ in $\mathcal{N}$ of infinite capacity for every $v \in V$ for which there is an $\operatorname{arc}(v, w)$ in $D_{n, p_{1}, \kappa}$ with tail $v$ and colour $x$. Finally, for each vertex $v \in V$ there is an $\operatorname{arc}(v, t)$ of capacity $d$.

For $S \subseteq C$, let $N(S)=\{v: x \in S, v \in V,(x, v) \in \mathcal{N}\}$ be the out-neighbour set of $S$ in $\mathcal{N}$. A cut of finite capacity can be obtained from a set $S \subseteq C$ and $N(S) \subseteq V$. Let $T=N(S), W=\{s\} \cup S \cup T$, and let $\bar{W}=(C \backslash S) \cup(V \backslash T) \cup\{t\}$. The capacity of the cut $(W: \bar{W})$ is $\kappa-|S|+d|T|$. Applying the max-flow min-cut theorem we see that $\mathcal{N}$ admits a flow of value $d n$ if and only if, for all $S \subseteq C$,

$$
\kappa-|S|+d|N(S)| \geq d n
$$

We estimate the probability that (1) is not true because, for some set $S,|N(S)|<$ $n-(\kappa-|S|) / d$. I.e. there exists a set of colours $S$ of size $s$ and a set of vertices $\bar{T}$ of size $|\bar{T}|>(\kappa-s) / d$ such that every arc of $D$ whose tail is in $\bar{T}$ has a colour in $C \backslash S$.

$p_{1}$ satisfies $1-p=\left(1-p_{1}\right)^{2}$ and so $p_{1} \geq p / 2$, for $1-\sqrt{1-p} \geq p / 2$ for $p \geq 0$. We see therefore that $n p_{1} \geq K_{0} \log n$.

Let $\mathcal{E}$ denote the subset of $D_{n, p_{1}}$ for which $\delta^{+}\left(D_{n, p_{1}}\right)>n p_{1} / 2$.

We first estimate $\operatorname{Pr}(\overline{\mathcal{E}})$. By the Chernoff inequality,

$$
\operatorname{Pr}\left(\delta^{+}\left(D_{n, p_{1}}\right) \leq n p_{1} / 2\right) \leq n e^{-n p_{1} / 8}=O\left(n^{1-K_{0} / 8}\right)
$$


which is $O\left(n^{-13 / 8}\right)$ for $K_{0} \geq 21$. Thus $\operatorname{Pr}(\overline{\mathcal{E}})=o(1)$

Let

$$
L(s)=2\left(\begin{array}{l}
\kappa \\
s
\end{array}\right)\left(\begin{array}{c}
n \\
\lceil(\kappa-s) / d\rceil
\end{array}\right)\left(\frac{\kappa-s}{\kappa}\right)^{(\kappa-s) n p_{1} /(2 d)}
$$

be an upper bound on the probability that some set of size $s$ does not satisfy (1) conditional on $\mathcal{E}$. The range of $s$ we need to consider is between $\kappa-d n+1$ and $\kappa-1$. For, if $|S|<\kappa-d n$ then (1) is true with $N(S)=\emptyset$, and if $s=\kappa$ then as $\delta^{+}(D) \geq n p_{1} / 2$, $\bar{T}=\emptyset$.

The probability that (1) is not satisfied is bounded by $\Theta$ where

$$
\Theta=\operatorname{Pr}(\overline{\mathcal{E}})+\sum_{s=\kappa-d n+1}^{\kappa-1} L(s)
$$

As $\operatorname{Pr}(\overline{\mathcal{E}})=o(1)$, we can concentrate on the summation term in (3).

Now, choosing $\kappa \geq 21 n$, and putting $\lceil(\kappa-s) / d\rceil=(\kappa-s) / d+f_{s}, 0 \leq f_{s}<1$,

$$
\begin{aligned}
\sum_{s=\kappa-d n+1}^{\kappa-1} L(s) & \leq 2 n e d \sum_{s=\kappa-d n+1}^{\kappa-1}\left(\frac{\kappa e}{\kappa-s}\left(\frac{n e d}{\kappa-s}\right)^{1 / d}\left(\frac{\kappa-s}{\kappa}\right)^{\left(K_{0} \log n\right) /(2 d)}\right)^{\kappa-s} \\
& \leq 2 n e d \sum_{s=\kappa-d n+1}^{\kappa-1}\left(3\left(\frac{\kappa-s}{\kappa}\right)^{K_{0} \log n /(2 d)-1 / d-1}\right)^{\kappa-s} \\
& \leq 2 n e d \sum_{s=\kappa-d n+1}^{\kappa-1}\left(3\left(\frac{d n-1}{e d n}\right)^{\left(K_{0} \log n\right) /(2 d)-1 / d-1}\right)^{\kappa-s} \\
& \leq 2 e d^{2} n^{2} \exp \left\{-\frac{K_{0} \log n}{2 d}+\frac{1}{d}+3\right\}
\end{aligned}
$$

which is $O\left(n^{-1 / 10}\right)$ for $K_{0} \geq 21$ when $d=5$.

Thus whp $\mathcal{N}$ contains a flow of value $n d$. The capacities of $\mathcal{N}$ are integral and so we can assume this flow is integral. It decomposes into $n d(s, t)$-paths, each of which assigns a colour $x$ to a vertex $v$. By construction a colour can be assigned at most once to an edge and each vertex is assigned $d$ colours. For each assignment of a colour $x$ to a vertex $v$ we choose (randomly from $D$ ) an arc of colour $x$ which has tail $v$. We thus obtain a multi-coloured member of $\mathcal{D}_{d-o u t, \kappa}$. It is easy to argue that the underlying uncoloured digraph is distributed as $D_{d-o u t, \kappa}$. Indeed we could start with $D_{n_{, p_{1}, \kappa}}$ and then replace each arc $(v, w)$ by $\left(v, \pi_{v}(w)\right)$ where the $\pi_{v}, v \in V$ are independent permutations of $V \backslash\{v\}$. After this transformation the digraph is still distributed as $D_{n, p_{1}, \kappa}$. We run the network flow algorithm and whp we obtain a multi-coloured member of $\mathcal{D}_{d-\text { out }, \kappa}$. 
By replacing each arc $(v, w)$ by $\left(v, \pi_{v}^{-1}(w)\right)$ we obtain a subgraph of the original $D_{n, p_{1}, \kappa}$ which is distributed as $D_{d-o u t, \kappa}$. For those cases where both $(v, w)$ and $(w, v)$ are selected by the algorithm to be edges of $D_{n, p_{1}, \kappa}$ we simply delete this edge. We have to do this because of the possibility that the network algorithm chooses a different colour for $\{v, w\}$ to the one chosen in going from $D_{n, p_{1}, \kappa}$ to $G_{n, p, \kappa}$.

In summary, whp $D_{n, p_{1}, \kappa}$ contains a multi-coloured subgraph which is distributed as $D_{d-\text { out }, \kappa}$. Ignoring orientation we obtain a graph which whp contains a Hamilton cycle. This verifies Theorem 1 for the case of undirected graphs.

Consider now the directed case i.e. we start with $D_{n, p, \kappa}$. We first split this into two independent copies $D_{1}, D_{2}$ of $D_{n, p_{1}, \kappa}$. We then use a slightly modified network. Now we have vertices $s, t, C$ and two copies $V_{1}, V_{2}$ of $V$. The $s, C$ edges are as before and there are $V_{1}, t$ and $V_{2}, t$ edges of capacity $d$ (now we can take $d=3$ ). We join $x \in C$ to $v \in V_{1}$ by an infinite capacity arc if $V_{1}$ contains an arc of colour $x$ and tail $v$. We join $x \in C$ to $v \in V_{2}$ by an infinite capacity arc if $V_{1}$ contains an arc of colour $x$ and head $v$. The network flow algorithm constructs a random multi-coloured 3-in,3-out digraph, which whp has a Hamilton cycle, by the result of [4], even after removing parallel arcs. This is why we take $d=3$ and appeal to the proof of the result in [2], that a random 3-in,3-out digraph is Hamiltonian whp. The proof there will survive the deletion of parallel arcs.

As a final remark, we did not really make arguments about Hamiltonicity only about constructing a random subgraph which is distributed as $D_{d-o u t, \kappa}$. Clearly, other monotone graph properties can be treated in this manner.

Finally we mention two natural related problems: Suppose we fix $K_{0}$ at the threshold value $\frac{1}{2}+o(1)$. What is the least value of $K_{1}=K_{1}(n)$ for which $G_{n, p, \kappa}$ contains a multi-coloured Hamilton cycle whp? Similarly, if we fix $K_{1}=1$, what is the least value of $K_{0}=K_{0}(n)$ for which $G_{n, p, \kappa}$ contains a multi-coloured Hamilton cycle whp? It is prudent to observe that we must take $K_{0} \geq 1+o(1)$ so that whp each colour occurs at least once.

\section{References}

[1] M. Albert, A.M. Frieze and B. Reed, Multicoloured Hamilton Cycles, Electronic Journal of Combinatorics 2 (1995) R10.

[2] C. Cooper and A.M. Frieze, Hamilton cycles in a class of random directed graphs, Journal of Combinatorial Theory B 62 (1994) 151-163

[3] C. Cooper and A.M. Frieze, Multicoloured Hamilton cycles in random graphs: an anti-Ramsey threshold, Electronic Journal of Combinatorics 2 (1995) R19. 
[4] C. Cooper and A.M. Frieze, Hamilton cycles in random graphs and directed graphs, Random Structures and Algorithms 16 (2000) 369-401.

[5] P. Erdös, J. Nesetril and V. Rödl, Some problems related to partitions of edges of a graph, in Graphs and Other Combinatorial Topics, Teubner, Leipzig (1983) 54-63.

[6] A.M. Frieze and T. Euczak, Hamiltonian cycles in a class of random graphs: one step further, in Proceedings of Random Graphs '87, Edited by M.Karonski, J.Jaworski and A.Rucinski, John Wiley and Sons (1990) 53-59.

[7] A.M. Frieze and B. D. McKay, Multicoloured trees in random graphs, Random Structures and Algorithms 5 (1994) 45-56.

[8] G. Hahn and C. Thomassen, Path and cycle sub-Ramsey numbers, and an edge colouring conjecture, Discrete Maths. 62 (1986) 29-33

[9] S. Janson and N.C. Wormald, Rainbow hamilton cycles in random regular graphs, to appear.

[10] R. Rue, Comment on [1]. Electronic Journal of Combinatorics 2 (1995) R10.

This research was sponsored in part by National Science Foundation (NSF) grant no. CCR-0122581. 